\title{
Direct-to-consumer clear aligner therapy comes to the UK
}

US teledentistry giant SmileDirectClub officially opened for business on 31 July to bring affordable and accessible clear aligner therapy to the UK. SmileDirectClub will open nine SmileShops in London, Manchester and Birmingham, with the first shops opening in Hammersmith, Oxford Circus and Tower Bridge in London, and Crossway in Birmingham.

SmileDirectClub will continue its rapid expansion in partnership with Well Pharmacy, who will pilot the opening of four additional SmileShops within Well Pharmacy locations in Cambridge, Newcastle, Cardiff, and Leeds.

SmileDirectClub has pioneered a unique teledentistry platform to connect customers with an affiliated network of UK-based registered dentists or orthodontists who direct all aspects of clinical care using SmileDirectClub's platform. These licensed dentists and orthodontists customise each patient's treatment plan and manage their patients' care from initial diagnosis through the conclusion of treatment, monitoring care along the way with remote check-ins every 90 days to allow patients to avoid the hassle of scheduling frequent visits to a dental practice.

SmileDirectClub has become one of the fastest-growing health technology companies in the US since launching in 2014, enabling 650,000 customers to 'get a smile they love', and has seen an uptake in its customers visiting their dentists more regularly to take better care of their teeth and to maintain their improved smile.

SmileDirectClub clear aligners are dentist-prescribed and custom-made from BPA-free plastic thermoformed onto personalised 3D-printed mouth moulds, powered by a groundbreaking fleet of HP industrial 3D printers. Each customer's clear aligner treatment plan is shipped in an 'all in one' box directly to the customer's door. As a result of the innovations across the supply chain, SmileDirectClub's

\section{Keep it incognito}

With the demands for more discreet treatments increasing, professionals need to find orthodontic appliances that can provide results as well as better aesthetics for patients during treatment.

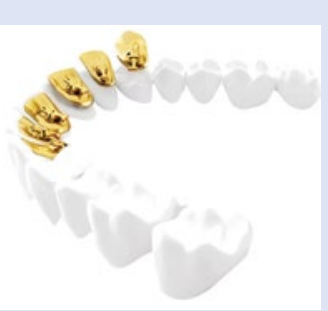

With the Incognito appliance system from 3M Oral Care, you can deliver near invisible treatment and achieve highly aesthetic results. As each Incognito appliance system features bespoke brackets, bonding trays and archwires, it can predictably achieve effective results

in a wide array of indications. Furthermore, as the appliance is placed behind the teeth, it can help patients retain confidence in their smile throughout treatment.

For more information, call 0845 8734066 or visit http://solutions.3m. co.uk/wps/portal/3M/en_GB/ orthodontics_EU/Unitek/.

$3 \mathrm{M}$ is a trademark of the $3 \mathrm{M}$ Company. Incognito is a trademark of TOP-Service für Lingualtechnik $\mathrm{GmbH}$.

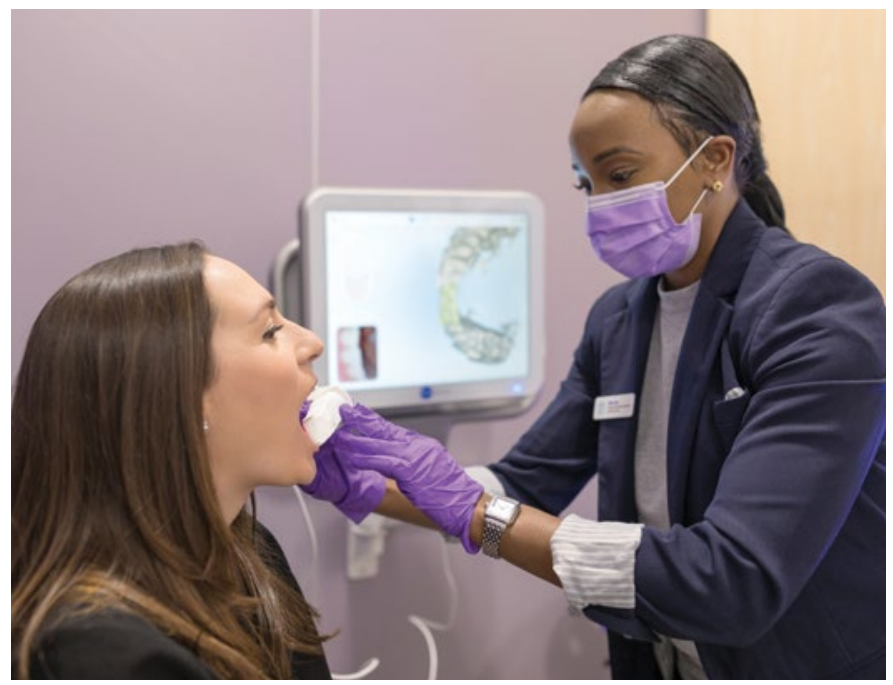

direct-to-consumer clear aligner therapy costs up to $60 \%$ less than traditional braces, with an average treatment length of six months, saving time and money for customers throughout the UK.

Customers can start their journey to a straighter smile at SmileDirectClub.co.uk, where they have the option to schedule a free visit to a SmileShop in their area to have 3D photographs captured of their smile, or they can order an impression kit, prescribed by a UK registered dentist or orthodontist. The treating dentist or orthodontist will create the custom treatment plan and prescribe custom-made, clear aligners, which are then shipped directly to the customer. SmileDirectClub offers two options for payment - a single payment of $£ 1,499$, or 12 payments of $£ 120$ per month with a deposit of $£ 199$, which includes two additional sets of retainers valued at $£ 70$ per set.

\section{Make one-appointment restorations a reality}

Want to offer your patients more by expanding your chairside restorative options?

With the CS 3100 milling system from Carestream Dental, you can make one-appointment restorations a reality.

Offering three

distinct fabrication options (grinding, carving and milling) the system can produce hybrid resin, ceramic and zirconia restorations that are ideal for single crowns, bridges, inlays, onlays, veneers and more.

The system also adapts to new materials with ease, meaning you can expand your restorative options further in the future.

For more information, contact Carestream Dental on 0800 1699692 or visit www.carestreamdental.co.uk. 